$>2.6-3.2$ (low DAS), $>3.2-5.0$ (moderate DAS), and $>5.1$ (high DAS), respectively. ${ }^{* *} p<0.01$, ${ }^{* * *} p<0.0001$ (Independent t-test and Kruskal Wallis test)

Acknowledgement: This study was supported by a research scholarship from the National Research Council of Thailand and by Mahidol University.

Disclosure of Interests: None declared

DOI: 10.1136/annrheumdis-2019-eular.1487

\section{SAT0008 IL-23 DRIVES PATHOGENIC TH17 CELLS THROUGH EPIGENETIC REGULATION BY STAT3 IN SLE PATIENTS}

Seunghyun Lee ${ }^{1}$, Shingo Nakayamada ${ }^{1}$, Satoshi Kubo ${ }^{1}$, Yamagata Kaoru ${ }^{1}$, Kitanaga Yukihiro ${ }^{1,2}$, Yoshiya Tanaka ${ }^{1} .{ }^{1}$ University of Occupational and Environmental Health, Japan, School of Medicine, Kitakyushu, Japan; ${ }^{2}$ Astellas Pharma Inc, Tokyo, Japan

Background: Systemic lupus erythematosus (SLE) is a systemic autoimmune disease characterized by loss of self-tolerance and by broad immune dysregulation, which result in overproduction of autoantibody and cause severe inflammation in various organs ${ }^{(1)}$. Although amplified mature Th17 cells are thought to be vital role in the development and progression of SLE, mechanism underlying their pathogenicity remain widely elusive in human $^{(2)}$.

Objectives: To elucidate the mechanism of pathogenic Th17 cells expansion, we probe the phenotype of Th17 cells in lupus patients and underlying epigenetic modification mediated by cytokine-induced signal transducer and activators of transcription (STAT) family factors in vitro. Methods: Human naive CD4+ T cells were cultured by Th17 polarizing condition (TGF- $\beta$, IL-6, and IL-1 $\beta$ ) for 5 days in vitro. To examine the role of specific cytokine in maturation of Th17 cells, we added various cytokines including IL-23 at the later time point (72hr). Expression of characteristic markers of pathogenic Th17 cell and STATs phosphorylation (p-STATs) were analyzed by flow cytometry and qPCR. The effect of baricitinib which is JAK1/JAK2 inhibitor on maturation of Th17 cell was investigated. Histone modifications were assessed by chromatin immunoprecipitation(ChIP)-PCR. To define the signals mediated by cytokine required for expansion of Th17 cell in SLE, Th17 phenotype and pSTATs were analyzed from blood samples of lupus patients by multi-color flow cytometry.

Results: In vitro, re-stimulation of Th17 cells with IL-23 markedly induced the characteristic markers of pathogenic Th17 cells such as ROR $\gamma \mathrm{t}$ and IL-17. IL-23 overwhelmingly activates the p-STAT3, not p-STAT4 for development of mature Th17 cells. IL-23-induced p-STAT3 was inhibited by baricitinib. In addition, proliferation of Th17 cells was suppressed by baricitinib in concentrate dependent manner. The loci of ROR $\gamma$ t at STAT binding sites were marked by bivalent histone modifications. After IL-23 stimulation, STAT3 exclusively bound on ROR $\gamma$ t gene loci supplemented by activating $\mathrm{H} 3 \mathrm{~K} 4 \mathrm{me} 3$ and repressing $\mathrm{H} 3 \mathrm{~K} 27 \mathrm{me} 3$ modifications. In memory Th17 cells, high proportion of IL-23R expression and STAT3/4 activation were identified from SLE patients compare with healthy individuals. Moreover, p-STAT3 was hypersensitively activated by IL-23 stimulation in memory Th17 cell only from lupus patients but not from healthy controls. Conclusion: This study proves that IL-23 serves as a pivotal factor that drives expansion of pathogenic Th17 cells. IL-23-mediated STAT3 alter histone modification, resulting in inflammatory function of pathogenic Th17 cell that are characteristically expanded in patients with SLE. These findings could be one of the underlying mechanisms of pathogenesis of SLE and helpful evidence toward novel therapeutic targets for SLE.

\section{REFERENCE}

[1] Tsokos GC. Systemic lupus erythematosus. N Engl J Med. 2011;365 (22):2110-21. (2) Ma, J., et al., The imbalance between regulatory and IL17-secreting CD4+ T cells in lupus patients. Clinical rheumatology, 2010. 29(11): p. 1251-1258.

Disclosure of Interests: Seughyun Lee: None declared, Shingo Nakayamada Grant/research support from: Mitsubishi-Tanabe, Takeda, Novartis and MSD, Speakers bureau: Bristol-Myers, Sanofi, Abbvie, Eisai, Eli Lilly, Chugai, Asahi-kasei and Pfizer, Satoshi Kubo Speakers bureau: BristolMyers, Pfizer, Takeda, and Eli Lilly, Yamagata Kaoru: None declared, Kitanaga Yukihiro Employee of: Astellas, Yoshiya Tanaka Grant/research support from: Abbvie, Astellas, Bristol-Myers Squibb, Chugai, Daiichi-Sankyo, Eisai, Mitsubishi-Tanabe, MSD, Ono, Taisho-Toyama, Takeda, Speakers bureau: Abbvie, Asahi-kasei, Astellas, Bristol-Myers Squibb, Chugai,
Daiichi-Sankyo, Eli Lilly, Eisai, Glaxo-Smithkline, Janssen, MitsubishiTanabe, Novartis, Pfizer Japan Inc, Sanofi, Takeda, UCB, YL Biologics DOI: 10.1136/annrheumdis-2019-eular.2923

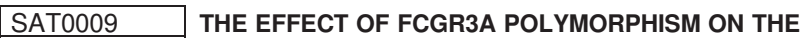 INITIAL DEPTH OF B-CELL DEPLETION BY RITUXIMAB, FUNCTIONAL NK-CELL MEDIATED KILLING AND CLINICAL RESPONSE IN SYSTEMIC LUPUS ERYTHEMATOSUS}

Md Yuzaiful Md Yusof ${ }^{1,2}$, James Robinson ${ }^{1}$,Yasser El-Sherbiny ${ }^{1}$, Andy C. Rawstron ${ }^{3}$, Paul Emery ${ }^{1,2}$, Ann Morgan ${ }^{1,2}$, Edward Vital ${ }^{1,2}{ }^{1}$ University of Leeds, Leeds Institute of Rheumatic and Musculoskeletal Medicine, Leeds, United Kingdom; ${ }^{2}$ Leeds Teaching Hospitals NHS Trust, NIHR Leeds Biomedical Research Centre, Leeds, United Kingdom; ${ }^{3}$ Leeds Teaching Hospitals NHS Trust, Haematological Malignancy Diagnostic Service, Leeds, United Kingdom

Background: Antibody-dependent cellular-mediated cytotoxicity (ADCC) is an important effector mechanism that contributes to the depletion of therapeutic antibody-opsonised cells in vivo. Rituximab (RTX) is an anti-CD20 antibody with a native IgG1 Fc, which crosslinks $F_{c}$ receptors (FcyRs) expressed on immune effector cells. NK cells, which express Fc $\gamma$ RIIla, are believed to play an important role with less well-characterised contribution from monocytes/macrophages and neutrophils. A genetic variant in FCGR3A (158V) with an increased affinity for lgG1 has been reported to correlate with clinical response to RTX in RA[1]. Data in SLE are limited as the FCGR genetic locus is characterised by structural variation making genotyping challenging.

Objectives: To assess the effect of FCGR3A-F158V polymorphism on (i) the initial depth of B-cell depletion by RTX; (ii) its functional effect on NK cell-mediated killing (iii) clinical response and (iv) 10-year RTX retention for the treatment of SLE.

Methods: A prospective longitudinal study was conducted in RTX-treated SLE patients in Leeds. B-cells were measured at baseline and 6 weeks using highly sensitive flow cytometry. Complete depletion was defined as total B-cell count $<0.0001 \times 10^{9} / \mathrm{L}$. A major clinical response $(\mathrm{MCR}$ ) was defined as improvement of all active BILAG-2004 domains to grade C/ better and no $A / B$ flare at 6 months. We measured qualitative polymorphism for FCGR3A using a multiplex ligation-dependent probe amplification as previously described[2]. Median expression of FcyRIIla on NK cells (CD3-CD56+CD16+) and NK cell degranulation (CD107a) in the presence of RTX-coated Raji cells (B-lineage cell line) were assessed using flow cytometry.

Results: 85 SLE patients with at least $2 \times$ BILAG B were studied [Female: $82(96 \%)$; mean age (SD): $40(14)$ years; $60 / 85(71 \%)$ on concomitant DMARDs; ANA positive: 100\%]. In cycle 1 RTX, 64/85(75\%) achieved BILAG response [major $=36 \%$; partial $=39 \%$ ]. Both complete depletion and MCR were associated with carriage of the FCGR3A-158V allele (158FV or 158VV), OR $2.73(95 \% \mathrm{Cl} 1.13-6.58)$ and $3.06(95 \% \mathrm{Cl} 1.19$ 7.58) vs $158 \mathrm{FF}$, respectively.

Patients who had complete B-cell depletion post-RTX had higher level of FcyRIIla on NK cells vs incomplete depletion; $p=0.006$. Comparing patients with FCGR3A-158V allele carriage vs FF genotype, there was a trend to higher expression of CD3-CD56+CD16+ in the former $p=0.073$ Moreover, degranulation as defined by ratio between\%CD107a+NK cell in the presence of Raji cells + RTX and\%CD107a+NK cell in Raji cells only, was higher in the $\mathrm{V}$ allele carriers vs FF genotype; $\mathrm{p}=0.024$.

Over 10-year follow-up, $27 / 85(31.8 \%)$ had stopped RTX [primary inefficacy $=7$; secondary inefficacy $=8$; $\mathrm{HACA}=10$; death=2]. FCGR3A-158FF genotype was associated with increased risk of RTX discontinuation; OR 2.36 (95\% Cl 1.08-5.18) after adjusting for age and DMARDs.

Conclusion: An ADCC-enhancing FcyRIlla variant was associated with initial complete B-cell depletion and MCR in RTX-treated SLE patients. This was supported by functional data on NK cell-mediated cytotoxicity and 10 year outcomes. These results elucidate one mechanism of resistance to rituximab in SLE and may guide development of more effective B-cell targeted strategies. With further validation, this polymorphism may be used to stratify SLE patients for therapy.

\section{REFERENCES}

[1] Ruyssen-Witrand A et al. ARD 2012

[2] Nagelkerke S et al. Genes and Immunity 2015

Acknowledgement: This research was funded by NIHR (DRF-2014 07 155). The views expressed are those of authors \& not necessarily the NHS, NIHR or DOH. 
Disclosure of Interests: Md Yuzaiful Md Yusof: None declared, James Robinson: None declared, Yasser El-Sherbiny: None declared, Andy C Rawstron: None declared, Paul Emery Grant/research support from: Pfizer, MSD, AbbVie, Bristol-Myers Squibb, Roche, Consultant for: Pfizer, MSD, AbbVie, Bristol-Myers Squibb, UCB, Roche, Novartis, Gilead,Samsung, Sandoz and Lilly, Ann Morgan: None declared, Edward Vital Grant/ research support from: He has received honoraria and research grant support from Roche, GSK and AstraZeneca.

DOI: 10.1136/annrheumdis-2019-eular.6919

\section{SAT0010 FUNCTIONAL BIOMARKER DEVELOPMENT FOR CELL- BASED THERAPY IN RHEUMATOID ARTHRITIS}

Maya Breitman ${ }^{1}$, Tracey Bonfield ${ }^{2}$, Susan Lasalvia ${ }^{1}$, Hillard Lazarus ${ }^{3}$, Arnold Caplan ${ }^{4}$, Nora Singer ${ }^{1} .{ }^{1}$ MetroHealth System at Case Western Reserve University, Medicine division of rheumatology, Cleveland, United States of America; ${ }^{2}$ Case Western Reserve University School of Medicine, Cleveland, United States of America; ${ }^{3}$ Case Western Reserve University School of Medicine, University Hospitals Cleveland Medical Center, Cleveland, United States of America; ${ }^{4}$ Case Western Reserve University School of Medicine, Skeletal Research Center, Cleveland, United States of America

Background: Rheumatoid Arthritis (RA) is an autoimmune disease in which early treatment prevents joint damage. Autoantibodies such as rheumatoid factors (RF) and anti-citrullinated peptide antibodies (APCA) define a subset of RA patients at the highest risk for damage. Reduction of RA disease activity is associated with improvement in function(s) of regulatory $\mathrm{T}$ cells (Treg) and attenuated responses of pro-inflammatory $\mathrm{T}$ effector cells (Teff). Human Mesenchymal Stem Cells (hMSC) isolated from bone marrow and culture-expanded have strong immunomodulatory properties; we hypothesize that therapeutic use of hMSCs may skew the immune system to resemble its pre-RA state. Lack of available biologic correlates measured early-on that indicate response to treatment limits efficacy assessment of cell-based therapy. Use of clinical response criterion requires large numbers of patients and may be impractical in early stage clinical trials. This work was performed in anticipation of conducting a Phase I safety trial of cell-based therapy in early RA.

Objectives: We set out to develop functional assay(s) as biomarkers of response that would indicate early-on that hMSC were or were not modulating immune function in a manner that could potentially predict potency and efficacy in RA patients.

Methods: Sero-positive female patients with active early RA (RAPID 3 avg 5.7/10 (high severity)) and healthy donor age and sex-matched controls were enrolled. Second passage bone marrow hMSCs were obtained from healthy donors $<30$ y.o. (Case Comprehensive Cancer Center Hematopoietic Biorepository and Cellular Therapy Core). Peripheral blood CD4+ $T$ cells were stimulated by cross-linking CD2/CD3/CD28 for 5 days. CD4+ T-cell proliferation rates were measured, and suppression indices calculated by comparing proliferation indices of CD4+ T-cells cultured with and without hMSC-conditioned medium.

Results: hMSC-related suppression $32.2 \pm 7.8$ (mean + - SE) percent in RA CD4+ T-cells compared to $45.7 \pm 8.9$ percent for healthy donors CD4 + T-cells. We demonstrated that soluble products from hMSCs can inhibit proliferative responses of CD4+ $T$ cells from patients with active RA though the studies were not powered to detect differences between RA and healthy donors. With limited number of early RA patients, correlation between level of MSC-related suppression and antibody status were not possible. That being said, the data trend appears promising.

Conclusion: Our ex vivo experiments demonstrated that MSCs may be suppressive in RA as well as in healthy donors. To our knowledge, this study is the first demonstration of the potential for using ex vivo suppression assays to predict response to cell-based therapy in early RA. Such assays might be performed to predict the efficacy of selected hMSCs to treat RA.

Acknowledgement: This study was supported by David and Virginia Baldwin Foundation, and by the Clinical and Translational Science Collaborative of Cleveland, 4UL1TR0002548 from the National Center for Advancing Translational Sciences (NCATS) component of the National Institutes of Health and NIH roadmap for Medical Research. Its contents are solely the responsibility of the authors and do not necessarily represent the official views of the $\mathrm{NIH}$.

Disclosure of Interests: None declared

DOI: 10.1136/annrheumdis-2019-eular.4998

\section{SAT0011 \\ TRANSCRIPTOMICS UNVEILS UNIQUE BIOLOGICAL PROFILE OF TERTIARY LYMPHOID STRUCTURES GERMINAL CENTERS}

Serena Colafrancesco ${ }^{1}$, Elena Pipi $^{2}$, Saba Nayar ${ }^{2}$, Joana Campos ${ }^{2}$,

Valentina lannizzotto ${ }^{2}$, Francesca Arienzo ${ }^{1}$, Roberta Priori ${ }^{1}$, Guido Valesini ${ }^{1}$,

Benjamin Fisher ${ }^{2}$, Francesca Barone ${ }^{2} .{ }^{1}$ Sapienza University of Rome, Rome, Italy;

${ }^{2}$ University of Birmingham, Birmingham, United Kingdom

Background: The development of the $B$ cell repertoire is regulated by the process of affinity maturation occurring within the inner part of the $B$ cell follicles [germinal centers (GCs)] within secondary lymphoid organs (SLOs). In autoimmunity, this process might occur within aberrant aggregates of lymphocytes in target organs, such as the ectopic lymphoid structures (ELS) found in the salivary glands of patients with Sjogren's Syndrome (SS) (1). The phonotypical and functional features supporting ELS which may sustain the development of autoimmune diseases have not been identified. Moreover, functional proof that ELS contribute to the development of autoimmunity independently from SLOs has not been provided.

Objectives: To characterise the transcriptome profile of human ELS isolated from SS salivary glands in comparison with SLOs.

Methods: Frozen minor salivary gland biopsies were obtained from SS patients and selected for presence of GCs+ ELS. Human tonsils were obtained by volunteers undergoing tonsillectomy for clinical need and used as SLO control. In order to detect GCs, all samples were stained for CD21, bcl6, CD20 and CD3. Sequential sections, were stained by Cresyl Violet and GCs (CD21+ infiltrates) from both salivary glands and tonsil were selectively microdissected (Laser Capture Microdissection). Salivary gland small infiltrates and large CD21-infiltrates were microdissected too. RNA was isolated and transcribed. RNA sequencing studies were performed using ClonTech SMARTseq v4 kit. Changes and differences in the expression of specific genes of interest were confirmed with targeted PCR studies.

Results: Transcriptomics analyses revealed that GCs from ELS and SLO exhibit markedly different gene expression profiles. Of note, sequencing unveiled that GCs from ELS are characterized by an aberrant cell-proliferation profile with downregulation of BCL6 and AID, the enzymes responsible for $B$ cell affinity maturation (both $p<0.0001$ ). Also in $E L S$, progressive upregulation of CD21 transcript mirrored increases in infiltrate size and organization (being lowest in small infiltrates and progressively higher in large CD21- infiltrates and CD21+ infiltrates, $\mathrm{p}=0.002$ ). Similar data were obtained for AID, whose expression was significantly higher in large CD21+ infiltrates compared to small infiltrates $(\mathrm{p}=0.0006)$. A cytokine signature was identified in ELS GCs based on the expression of TNF, INFY, BAFF, APRIL, CXCL12, CXCL13, FAS, and FASL, all of which were upregulated compared to GCs from tonsils.

Conclusion: Our studies reveal that GCs forming in ELS exhibit marked transcriptional differences from classic GCs observed in SLOs. Main features characterizing ELS include lower levels of Bcl6 and AID, aberrant apoptosis, and a pathogenic inflammatory cytokine signature. Despite similarities in the anatomical organization and appearance of GCs in ELS and SLO, critical transcriptional differences emerge, which are likely functionally implicated in impaired regulation of the B cell cycle and survival of autoreactive B cell clones, ultimately leading to the development of autoimmune disease.

\section{REFERENCE}

[1] Barone F. et al. PNAS 2015.

Disclosure of Interests: Serena Colafrancesco: None declared, Elena Pipi: None declared, Saba Nayar: None declared, Joana Campos: None declared, Valentina lannizzotto: None declared, Francesca Arienzo: None declared, Roberta Priori: None declared, Guido Valesini: None declared, Benjamin Fisher: None declared, Francesca Barone Grant/research support from: GlaxoSmithKline, Roche, UCB Pharma, Actelion, ONO Pharmaceutical, Consultant for: GlaxoSmithKline, Roche, Actelion, ONO Pharmaceutical

DOI: 10.1136/annrheumdis-2019-eular.6993 